\title{
Metrology Needs for Metal Additive Manufacturing Powders
}

\author{
JOHN A. SLOTWINSKI ${ }^{1,3}$ and EDWARD J. GARBOCZI ${ }^{2}$ \\ 1.-The Johns Hopkins University Applied Physics Laboratory, Laurel, MD, USA. 2.-Applied \\ Chemicals and Materials Division, National Institute of Standards and Technology, Boulder, CO, \\ USA. 3.-e-mail: john.slotwinski@jhuapl.edu
}

\begin{abstract}
Additive manufacturing (AM) processes can produce highly complex and customized parts without the need for dedicated tooling and can produce parts directly from the part design information. These types of processes are poised to revolutionize the manufacturing industry, yet several challenges are currently preventing more widespread adoption of AM technologies. Among these challenges are metrology issues associated with the measurement and characterization of the metal powders used for AM systems. This article will describe the technical challenges and needs for characterizing metal AM powders, recent research efforts to address those needs, and current work to standardize characterization methods in ASTM and ISO, such as the recently released ASTM F3049, Standard Guide for Characterizing Properties of Metal Powders Used for Additive Manufacturing Processes.
\end{abstract}

\section{INTRODUCTION}

Unlike conventional manufacturing subtractive processes, in which a tool is used to remove unwanted material from a work piece to fabricate a part, additive manufacturing (AM) is "a process of joining materials to make objects from $3 \mathrm{D}$ model data, usually layer upon layer." ${ }^{\text {These processes }}$ have several advantages that distinguish them from traditional removal processes and make them suitable for complex and high-value parts:

- Parts with complex geometries, internal features, engineered porosity, and material gradients can be realized.

- High levels of customization make AM suitable for small lot sizes (even lot sizes of one).

- One can go directly from digital design to manufactured part.

- No tooling, fixtures, or path planning are required, making it quick and inexpensive to incorporate part customization and design changes.

- The process is suitable for hard metal materials that traditionally are difficult to machine efficiently with a removal process.

In recent years, AM has received significant attention in both the popular press and in scientific journals; in fact, from 2011 to 2012 the number of publications on AM jumped by an order of magnitude, from 1600 to $16,000 .^{2}$ There have also been several high-publicity examples of AM, such as a metal jaw replacement ${ }^{3}$ and the announcement of plans by an aircraft engine manufacturer to incorporate an additively-made metal fuel nozzle into an engine. ${ }^{4}$ These application examples have helped fuel the AM fervor that is common in the popular press. However, in some cases it is difficult to distinguish between the vision of AM and the hype, ${ }^{5}$ and while predicting the actual future impact of AM is difficult, it is easy to imagine how AM could potentially impact every major industry that it touches. ${ }^{6}$

Many technical challenges and hurdles have been identified via collaborative road-mapping efforts, ${ }^{7,8}$ which prevent the full vision of AM from being realized today. Chief among these hurdles are a litany of materials-related issues that must be solved, including issues associated with the metal powders that are used as input material for some AM systems. This article will describe the technical challenges and needs for characterizing metal AM powders, some recent examples of research efforts to address those needs, and current work to standardize characterization methods in the ASTM International (ASTM) and the International Organization for Standardization (ISO), such as the recently released ASTM F3049, Standard Guide for Characterizing Properties of Metal Powders Used for Additive Manufacturing Processes. ${ }^{9}$ 


\section{TECHNICAL CHALLENGES FOR METAL POWDERS}

In the United States, there have been two conspicuous road-mapping efforts in the last 5 years, one sponsored by the National Science Foundation (NSF) ${ }^{7}$ the other by the Department of Commerce's National Institute of Standards and Technology (NIST) ${ }^{8}$ which help define the technical challenges that are delaying more widespread adoption of AM technologies. Both of these included participation by industry, academia, and government agencies. The NIST effort ${ }^{8}$ was focused primarily on needs associated with metals-based AM systems, and it outlined broad industry strategies for addressing those technical challenges.

A common theme in both reports is the many challenges associated with AM materials. Some of the technical needs associated with AM materials include:

- It must be understood that the relationship between material properties and powder properties is limited.

- The underlying factors responsible for machineto-machine or day-to-day variability are not completely understood.

- Greater understanding and characterization of AM materials is needed, including input material characteristics such as powder size, shape, and chemistry.

- Standardized methods for qualifying and certifying AM parts and AM materials are lacking or are inadequate and traditional methods for material qualification and certification of AM materials are largely impractical in time, effort, and cost (See also, for example, Ref. 10).

- Qualification of material feedstock, including metal powders, is limited, and there is a lack of understanding of the differences between virgin and used metal powder.

- AM material data, both general data, and highquality, pedigreed, traceable data that are necessary for design-allowable databases, are lacking.

- No standardized AM-specific methods for conducting AM material interlaboratory studies exist.

Included in this partial list are issues associated with the metal powders used in AM. These powders are most commonly produced by gas atomization, are typically tens of micrometers in size, and have morphologies that are generally spherical, although a given batch of powder may include many instances of nonspherical or quasi-spherical morphologies. ${ }^{11}$ Figure 1 shows a representative scanning electron microscopy (SEM) image of stainless steel powder with these typical morphologies. A given batch of powder, particularly for AM powderbed systems, needs to have a distribution of sizes so as to allow for better packing (e.g., higher packing densities) if it is to be used in a powder-bed AM system. These distributions are not always rigorously optimized for a given powder, and the interaction between this size distribution and a given AM process has not been quantified and could have significant effects. ${ }^{11}$

Recent scientific papers have also discussed more specific technical issues associated with AM metal powders. For example, Clayton ${ }^{12}$ showed the importance of generating powder data that are relevant to the additive process that is using the powder and how critical it is to not rely on a single measured powder characteristic. It has been shown that two powders that have the same measured shear cell behavior (which measures the powder's shear stress at different applied normal stresses) could have markedly different dynamic behavior, and the dynamic behavior, which is not a standard practice in measuring AM powders, may be more representative of the powder flow in an AM system. Certainly, one conclusion could be that the currently employed methods for measuring AM powders are not sensitive enough or appropriate to fully capture the powder characteristics that are important to an AM process. Another conclusion is that best practice should incorporate a combination of shear, dynamic, and bulk property measurements if one is to fully understand powder flow behavior in AM systems.

Lyckfeldt $^{13}$ described opportunities for better measuring the characteristics of metal AM powders. He showed how different batches of AM steel powders, despite having similar particle size distributions, can have significantly different flow properties, which can affect their performance in a metal AM system. Measurement methods using Hall flowmeters, which are a common way for characterizing metal powders, also have limitations, and particle properties such as morphology, surface roughness, surface chemistry, size distribution, and

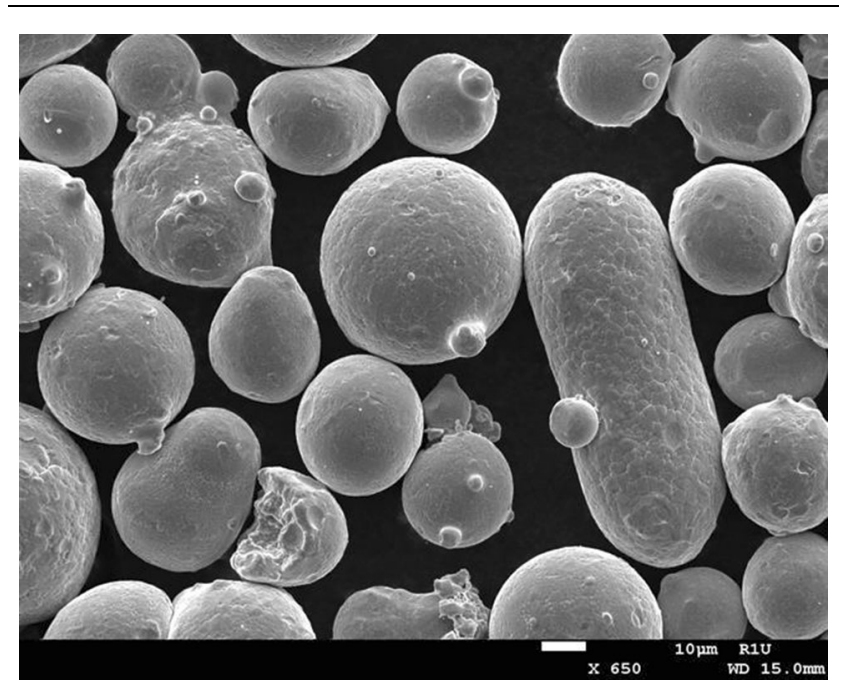

Fig. 1. Representative SEM image of steel AM powder showing typical spherical and nonspherical morphologies. The white scale bar represents $10 \mu \mathrm{m}$ and the image is roughly $170 \mu \mathrm{m}$ wide. 
environmental conditions can affect the measurement results. Because of this, advanced powder rheometry methods, which have not historically been employed in a widespread way for AM powders, may be necessary for discerning powder flow differences. ${ }^{13}$

In the area of size distribution measurements of a batch of particulates, historically laser diffraction measurements have been used extensively. These techniques, typically realized in a commercial instrument, quickly measure the size of particles based on the diffraction pattern from a laser beam that passes through the particles. The AM industry has also used these instruments for measuring the size distribution of metal powders. These methods have been found, however, to have limitations.

For example, this method assumes spherical particles $^{14}$ and is less accurate for particles with nonspherical morphologies, ${ }^{14}$ which are definitely present in metal AM powders. ${ }^{11}$ Over a range of particle diameters from $12 \mu \mathrm{m}$ to $64 \mu \mathrm{m}$, which is representative of many AM metal powders, the measurement uncertainties range from $1.5 \%$ to $9 \%$, for both wet and dry methods, where the particles are dispersed in a liquid or aerosol, respectively. ${ }^{14}$ There are other limitations as well. The assumption of having sufficient randomization in the particles' orientation has been shown not to be true because the flow in these devices is typically laminar, and not turbulent, resulting in measurement artifacts such as having two peaks (where there should only be one) in the measured size distribution. ${ }^{14}$ Sharp edges on the particles can also give diffraction "ghosts," which are interpreted as small particles and may skew the measurement results toward smaller particles. ${ }^{14}$ Finally, the complex refractive indices of both the sample and the liquid suspension are needed to get the correct measured results. ${ }^{14}$ All of these issues affect the accuracy of the laser diffraction method and hence limit the ability to accurately characterize metal AM powders.

Other methods for powder characterization also have limitations. For example, Hall funnels ${ }^{15}$ can be used to measure a powder's coefficient of friction by measuring the angle of repose of powder deposited into a pile. However, it has been shown that this method may not be sensitive enough or accurate enough to measure fine differences in the coefficient of friction. ${ }^{16}$ Understanding a powder's friction is important to understanding how it flows and packs in a powder-bed AM system, and thus it is related to the final characteristics of the part being made because better packing generally results in better final material characteristics, such as less porosity or more uniform monosize pores.

Finally, it is commonly known that environmental factors such as relative humidity can have an effect on powder measurements, especially in measurements of bulk density and flow. In cases of high humidity, the powder may end up clumped together, which then affects its packing and ability to flow.
Laboratory methods for measuring powder density, including both bulk density and tap density, also are not representative of the true powder packing density of a powder in an AM system's powder bed, especially after the powder has been spread. The true density is critically important because it is needed as an input into high-fidelity physics-based models of AM processes (see for example Ref. 17). Currently, some nascent work is underway to develop measurement methods to measure the true in situ powder-bed density and packing. ${ }^{18}$

The above attempts to illustrate some of the technical challenges, including measurement methods associated with metal AM powders. The limitations in the applicability and sensitivity of these methods prevent them from fully discerning the qualities of and differences found in metal AM powders. Fortunately, there exists a portfolio of research and development work that is focused on metal AM powders, which may provide the foundation needed for improving these methods.

\section{EXAMPLES OF CURRENT POWDER RESEARCH}

The number of AM powder research projects has grown rapidly in the last few years, a reflection of the industry's realization of the importance of fully understanding powder properties. What follows are some examples of this research. It is not meant to be comprehensive, but rather it is illustrative of some different kinds of work that are currently going on in industry, the government, and academia, which shows the promise of embodying the research foundation for the progress that is needed.

For example, Starr et al. ${ }^{19}$ showed that powders have different microstructures depending on the gas used in the gas atomization and that the powder microstructure, and not the inert gas used in the AM process, is primarily responsible for the microstructure of the final part. In this work, nominally identical stainless steel powders with size distributions and morphologies that were appropriate for AM were obtained from two different commercial sources. One of these powders was made via gas atomization in a nitrogen environment, and the other via gas atomization in an argon environment. X-ray diffraction measurements showed that the phase composition of the nitrogen-atomized powder was mostly austenitic, while the phase composition of the argon-atomized powder was mostly martensitic. These powders were used to make mechanical test specimens in a laser-based powder-bed fusion AM system in both nitrogen and argon build environments. It was found that, independent of the build atmosphere used, the argon-atomized powder resulted in additively-built material that was nearly all martensitic. Conversely, the nitrogen-atomized powder resulted in additively-build material that was nearly completely devoid of the martensitic phase. These results show the importance of 
knowing the processes parameters used in making AM powders and highlight that more research and development is needed to better understand the relationship between input powder properties and part properties.

It is instructive to compare Starr et al. ${ }^{19}$ work to that of Murr et al. ${ }^{20}$ who performed a similar set of experiments. They also used steel powders that were gas atomized in both argon and nitrogen, and they performed builds in both nitrogen and argon environments using a commercial laser-based powder-bed AM system that was nominally identical (same vendor and model) to that of Starr et al. ${ }^{19}$ The final phase results were similar except for the case of powder that was gas atomized in nitrogen and processed in argon, which Murr et al. ${ }^{20}$ found to have a martensitic phase (Starr et al. ${ }^{19}$ found it to be primarily austenitic). This difference might be explained by the different chemical compositions of the steel powder (Starr's powder was $17 \% \mathrm{Ni}$ and $4 \%$ Cr, while Murr's powder was 3\% to 5\% Ni and 15\% to $17.5 \% \mathrm{Cr}$ ). Another possible explanation for the difference might be the post-build heat treatments, which in the Murr study were done in an argon environment at $482^{\circ} \mathrm{C}$ for $1 \mathrm{~h}$. While Starr found that heat treating his austenitic specimens at lower than $700^{\circ} \mathrm{C}$ did not change the material phase, this lack of transformation might again be due to the differing initial powder composition. Regardless, this further emphasizes the point that the initial powder properties must be well understood because there is a linkage between powder properties and the final part properties.

NIST recently published a study ${ }^{11}$ on AM metal powder characterization using an extensive array of characterization techniques applied to both virgin (stainless steel and cobalt chrome powders) and recycled (stainless steel powder) states. The AM process used was direct metal laser melting (DMLM). The physical techniques included laser diffraction (LD) particle size analysis, x-ray computed tomography (CT) for size and shape analysis, helium pycnometry for powder density, and optical and SEM for particle morphology. X-ray diffraction (XRD) and x-ray photoelectron spectroscopy (XPS) were techniques sensitive to surface chemistry and were also employed. The purpose of the study was not to advocate using these sophisticated powder characterization techniques in the industrial environment as a substitute for traditional techniques but rather to discover the parameters that are important for any proposed characterization technique to measure.

The results found for virgin powder results were interesting. For both metal powders, a bucket-tobucket consistency was found, indicating consistency across the production lot, considering size distribution, particle shape, chemistry (phase information), and density averaged over many particles per bucket. The virgin cobalt chrome powder included more small particles than did the virgin stainless steel powder, as shown in both by direct SEM imaging and the particle size distribution (PSD) as measured by laser diffraction PSD, with the average size being smaller for the cobalt chrome. The particles were found to be roughly spherical but with important nonsphericities due to fracture, satellites, joined particles, and different intrinsic morphology (e.g., "tear-drop" shape), seen in SEM imaging and quantitatively illustrated using x-ray CT shape distribution functions made from shape data for thousands of particles. In the stainless steel powder, the predominant crystallographic phase found by XRD was face-centered cubic (fcc) austenite, with a minor body-centered cubic (bcc) component. The main cobalt chrome component was fcc, with little trace of any other kind of crystal structure.

The stainless steel powder PSD generally skewed somewhat larger as the number of recycling cycles increased because particles much smaller than the usual sieve opening employed $(80 \mu \mathrm{m})$ could fuse together yet still be passed through the sieve in the recycling process. XRD showed that there were also small changes in the proportion between the fcc and bcc phases in the stainless steel particle surfaces as the number of recycling cycles increased, while XPS did not show any surface differences. In looking at the sieve residue, however, XPS showed clear chemical differences on the surfaces of the stainless steel sieve residue particles versus the virgin particles. SEM images of the stainless steel powder in the sieve residue showed melting and particle joining (Fig. 2). X-ray diffraction found a large change in composition, with the fcc and bcc phases almost equal in volume fraction. Finally, PSD differences, as measured both by laser diffraction and by x-ray CT, from recycled samples taken before and after sieving, showed that the powder spreader arm in the AM machine used preferentially transported larger particles $(+60 \mu \mathrm{m})$ past the build plate, which were then not incorporated into the build. Hence, careful particle size measurements were able to give insight into the manufacturing process used.

\section{STANDARDIZATION EFFORTS}

An additional challenge to more widespread proliferation of metal AM technologies is a lack of consensus standards for AM. ${ }^{7,8}$ Standards are tools that ultimately provide the user the confidence that various measurement methods, specifications, practices, and terms are being used correctly and consistently. ${ }^{21}$ The industry already has AM standardization efforts in two international standards development organizations (SDOs), ASTM and ISO. The respective AM committees are ASTM F42: Additive Manufacturing Technologies ${ }^{22}$ and ISO Technical Committee (TC) 261: Additive Manufacturing. ${ }^{23}$ Fortunately, the environment for the development of AM standards is favorable because (I) AM technology development is at an early stage, 


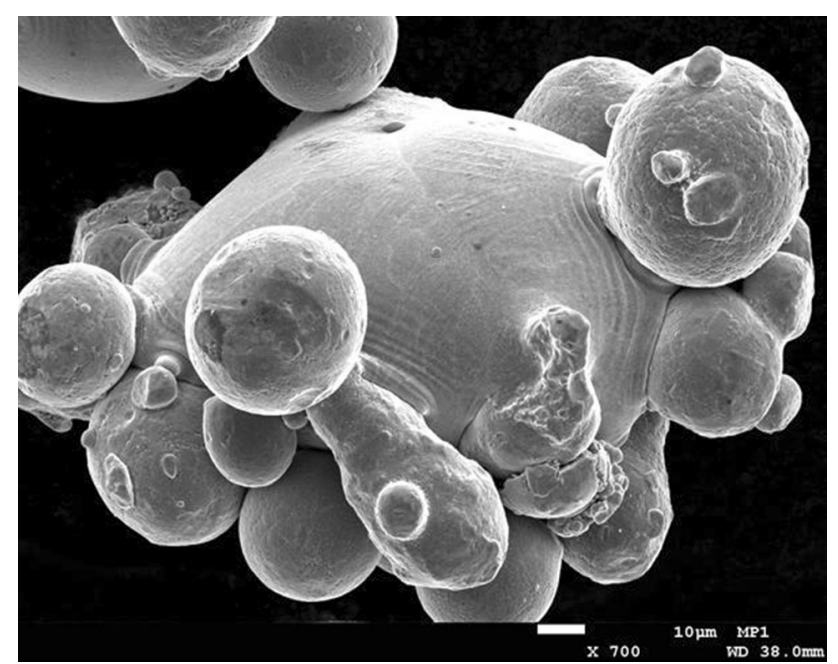

Fig. 2. View of multiple stainless steel residue particles that are clearly bonded by a melt phase. The white bar represents a length of $10 \mu \mathrm{m}$.

(II) ASTM-I F42 and ISO TC261 have an advantageous formal working agreement (see below), and (III) the AM research and standard community is relatively small, resulting in efficient exchanges of information in a spirit of collaboration.

ASTM F42 was organized in January 2009 by the AM industry, and currently it has a roster of more than 200 individuals and organizations with representation from at least 16 countries. $^{24}$ The committee meets twice a year, alternating between locations in the United States and Europe, to serve its international composition. It is composed of five technical subcommittees, with oversight provided by an executive committee. Currently, ASTM F42 has 10 approved standards and roughly 20 work items for proposed new standards under development. ${ }^{25}$ F42 also has established a strategic plan for standards development that identifies those areas where future standards are needed, including highlevel, specific, and specialized standards. ${ }^{26}$ Some of these standards, particularly those for measuring the mechanical properties of AM specimens and the properties of AM powders, will be based on existing standard test methods in the mechanical property testing and powder metallurgy industries, although some modification of these methods may be necessary to account for the unique characteristics of and needs for AM materials.

ASTM F42 and ISO TC 261 have a formal working agreement ${ }^{27}$ that is unique among cooperating standards development organizations. Signed in September 2011 by the ISO Secretary General and the ASTM President, this agreement facilitates: (I) fast track processing of existing ASTM standards by the ISO as Draft International Standards, (II) adoption of ISO standards by ASTM, and (III) co-development of joint ISO/ASTM standards. To date, two standards have dual ISO/ASTM designations and several ISO/ASTM task groups are working on joint standards. This favorable arrangement will minimize the need for future standards harmonization of similar but different standards that reside in distinct SDOs, which can be a long and painful process. This SDO cooperation will lead to a greater proliferation of AM technologies.

ASTM F3049 is a high-level standard for metal AM powders that was approved as a formal standard in $2014^{9,28}$ and is the first powder-specific standard developed and released by ASTM F42. It identifies 37 existing ASTM, ISO, and Metal Powder Industries Federation (MPIF) standardized powder metal metrology methods that may be useful for AM powders and used a recent NIST Internal Report as its basis. ${ }^{29}$ These referenced standards include methods for correct powder sampling and methods for measuring powder size, morphology, chemical composition, flow, and density. In the words of the standard itself:

The intention for this guide is to provide purchasers, vendors, or producers of metal powder to be used in additive manufacturing processes with a reference for existing standards or variations of existing standards that may be used to characterize properties of metal powders used for additive manufacturing processes. It will serve as a starting point for the future development of a suite of specific standard test methods that will address each individual property or property type that is important to the performance of metal-based additive manufacturing systems and the components produced by them. While the focus of this standard is on metal powder, some of the referenced methods may also be appropriate for non-metal powders. ${ }^{9}$

This standard is "one-stop-shopping" in that it is a single document that points the reader to existing methods for powder characterization, and as such it is especially useful for educating those who are new to metal-based AM. The standard does not make any judgments on the applicability of these standards for AM powders because the referenced standards were not originally developed with AM in mind, but it is only a first step to the standard that is ultimately needed. NISTIR 8005, Applicability of Existing Materials Testing Standards for Additive Manufacturing Materials, makes some judgment on the applicability of these existing methods for AM powders, but the assessment is based only on the authors' knowledge and experience with the various test methods and metal powder-bed AM systems, and as such may not be comprehensive. ${ }^{30}$

What is next for AM powder standards in the ASTM F42 committee? Now that a general high-level standard for measuring metal AM powders is in place, more specific, lower-level standards will be developed. Like F3049, these standards will build on existing powder metrology methods but tailor them for AM powders. For example, as one possibility, we have shown that existing and com- 
monly used methods for particle size distribution measurements (via laser diffraction) ${ }^{11}$ and flow (via Hall flow-meters $)^{16}$ have limitations. These methods may require enhancement, or the development of more sophisticated measurement tools to discern subtle differences in powders, if these subtle differences are important to a specific AM process. Since the development of consensus-based standards is driven by the needs and interests of the SDO members, the AM community will ultimately prioritize the technical needs that are most important to them, and hence determine the order of standards developed.

\section{CONCLUSION}

Metal-based AM is poised to be a manufacturing technology that positively affects every major industry in the world. The vision of metals-based $\mathrm{AM}$, and the resulting applications that use the revolutionary parts made via $\mathrm{AM}$, is inspirational. However, this vision is not fully a reality today due to several technical challenges, including challenges associated with the measurement of AM metal powders and with the relationship between powder properties and part properties. Fortunately, a portfolio of research and development being undertaken by industry, academia, and the government will help overcome these challenges and fulfill the vision. There are also standardization efforts, including the recent release of ASTM F3049, the first AM powder-specific standard, which will lead to greater proliferation of AM technologies.

\section{REFERENCES}

1. ASTM F2792-12, Standard Terminology for Additive Manufacturing Technologies (2012).

2. T. Wohlers, Wohler's Report 2012: Additive Manufacturing and $3 D$ Printing State of the Industry (Fort Collins, CO: Wohlers Associates Inc., 2012).

3. BBC, Transplant jaw made by 3D printer claimed as first. (2012), http://www.bbc.co.uk/news/technology-16907104.

4. GE Reports, Fit to print: New plant will assemble world's first passenger jet engine with 3D printed fuel nozzles, nextgen materials. (2014), http://www.gereports.com/post/ 80701924024/fit-to-print.

5. T. Wohlers and T. Caffrey, Additive manufacturing: Going mainstream. (2013), https://www1.eere.energy.gov/manu facturing/pdfs/sme_man_engineering.pdf.

6. J. Hsu, Why 3D printing matters for "made in USA," TechNewsDaily (December 2012).

7. D.L. Bourell, M.C. Leu, and D.W. Rosen, eds., Roadmap for Additive Manufacturing Identifying the Future of Freeform Processing (Austin, TX: The University of Texas at Austin Laboratory for Freeform Fabrication Advanced Manufacturing Center, 2009), http://wohlersassociates.com/road map2009.html.

8. Energetics Incorporated, ed., Measurement Science Roadmap for Metal-Based Additive Manufacturing Workshop Summary Report (Columbia, MD: Energetics Incorporated, 2013), http://events.energetics.com/NIST-AdditiveMfgWork shop/pdfs/NISTAdd_Mfg_Report_FINAL.pdf.

9. ASTM F3049-14, Standard Guide for Characterizing Properties of Metal Powders Used for Additive Manufacturing Processes (2014).
10. C. Brice, Proceedings of the 1st World Congress on Integrated Computational Materials Engineering (ICME) (Hoboken, NJ: John Wiley \& Sons Inc., 2011).

11. J.A. Slotwinski, E.J. Garboczi, P.E. Stutzman, C.F. Ferraris, S.S. Watson, and M.A. Peltz, NIST J. Res. (2014). doi:10. 6028/jres.119.018.

12. J. Clayton, Characterising powders to optimise additive manufacturing. $t c t, 6$ (June 2014), http://www.tctmagazine. com/additive-manufacturing/characterising-powders-to-opti mise-additive-manufacturing/.

13. O. Lyckfeldt, Proceedings of the Euro PM2013 Congress \& Exhibition, Vol. 2 (Shrewsbury, U.K.: European Powder Metallurgy Association, 2013), pp. 225-230.

14. C.F. Ferraris, W. Guthrie, A.I. Aviles, M.A. Peltz, R. Haupt, and B.S. MacDonald, Certification of SRM 114q: Part II (Particle Size Distribution), NIST Special Publication 260166 (Gaithersburg, MD: NIST, 2006).

15. ASTM, B213-11, Standard Test Methods for Flow Rate of Metal Powders Using the Hall Flowmeter Funnel (2011).

16. H. Mazhar, J. Bollmann, E. Forti, A. Praeger, T. Osswald, and D. Negrut, Technical Report TR-2013-3 Studying the Effect of Powder Geometry on the Selective Laser Sintering Process, unpublished research, 2014, http://sbel.wisc.edu/ documents/TR-2013-03.pdf.

17. N. Patil, D. Pal, and B. Stucker, A New Finite Element Solver Using Numerical Eigen Modes for Fast Simulation of Additive Manufacturing Processes," International Solid Freeform Fabrication Symposium Proceedings (University of Texas at Austin, 2013).

18. Private communication with NIST Intelligent Systems Division (2014).

19. T.L. Starr, K. Rafi, B. Stucker, and C.M. Scherzer, International Solid Freeform Fabrication Symposium Proceedings (Austin, TX: University of Texas at Austin, 2012).

20. L.E. Murr, E. Martinez, J. Hernandez, S. Collins, K.N. Amato, S.M. Gaytan, and P.W. Shindo, J. Mater. Res. Technol. 1, 167 (2012).

21. J.A. Slotwinski, Additive manufacturing standards for metallic materials. Technology Exchange on Metallic Materials for Additive Manufacturing, November 5, 2013 (State College, PA: Penn State University, 2013).

22. Committee F42 on Additive Manufacturing Technologies, http://www.astm.org/COMMITTEE/F42.htm.

23. ISO/TC 261, Additive Manufacturing, http://www.iso.org/ iso/standards_development/technical_committees/other_bod ies/iso_technical_committee.htm?commid=629086.

24. ASTM International, Fact Sheet on ASTM Technical Committee F42 on Additive Manufacturing Technologies, http:// www.astm.org/COMMIT/F42\%20Fact\%20Sheet\%202014. pdf.

25. Subcommittees in Committee F42 on Additive Manufacturing Technologies, http://www.astm.org/COMMIT/SUB COMMIT/F42.htm.

26. ISO/TC 261 and ASTM F42, Joint Plan for Additive Manufacturing Standards Development, http://www.astm.org/ COMMIT/AM_Standards_Development_Plan_v2.docx.

27. ASTM International, News Release: "Additive Manufacturing to Benefit from Standards Agreement," http://www. astmnewsroom.org/default.aspx?pageid=2608.

28. ASTM International, News Release: "Properties of Metal Powders Used for Additive Manufacturing Described in New ASTM Standard," http://www.astmnewsroom.org/default.as px? pageid $=3490$.

29. A.L. Cooke and J.A. Slotwinski, Properties of Metal Powders for Additive Manufacturing: A Review of the State of the Art of Metal Powder Property Testing, NIST IR 7873 (Gaithersburg, MD: NIST, 2012), http://dx.doi.org/10.6028/ NIST.IR.7873.

30. J.A. Slotwinski and S.P. Moylan, Applicability of Existing Materials Testing Standards for Additive Manufacturing Materials, NIST IR 8005 (Gaithersburg, MD: NIST, 2014). doi: 10.6028/NIST.IR.8005. 